\title{
Using in situ density and strength measurements for sediment maintenance in ports and waterways
}

\author{
Alex Kirichek $^{1,2}$ (D) Ahmad Shakeel ${ }^{2,3} \cdot$ Claire Chassagne $^{2}$ \\ Received: 7 June 2019 / Accepted: 3 February 2020 / Published online: 19 February 2020 \\ (C) The Author(s) 2020
}

\begin{abstract}
Purpose Fluid mud layers can be substantial in waterways and they can jeopardise navigation when the ship's keel comes in its vicinity. The nautical bottom has therefore to be properly characterised. Mud density and yield stress are used as criteria to characterise the nautical bottom. For a decade, measuring these parameters in situ has been a challenging task. The goal of this paper is to compare the parameters obtained from recently developed in situ measuring instruments.

Materials and methods Two vertical profilers (Graviprobe and Rheotune) were used in this study. The Graviprobe measures the undrained shear strength, whereas the Rheotune measures the Bingham yield stress and density of mud layers. Four different locations at the Port of Hamburg were used to test the Rheotune. Bingham yield stresses obtained from Rheotune measurements were compared with the laboratory rheological measurements. The mud layer thicknesses profiled by Graviprobe and Rheotune at the Port of Hamburg and at the Port of Rotterdam were compared. The measurements were also compared with a model that predicts the consolidation of weak mud.

Results and discussion The Rheotune data match well with the laboratory experiments at two of the four tested locations at the Port of Hamburg. For the other two locations, the Rheotune data underestimates the Bingham yield stress, as compared with the laboratory data. The reason for the discrepancy is discussed. The comparison between the Graviprobe's and Rheotune's profiles showed that the thickness of the fluid mud layer, found from each device, for all locations, is similar. The in situ density measurements were confirmed by the laboratory experiments. It was found that the standard model for consolidation of mud could reproduce the in situ strength and density measurements as a function of time relatively well. The mismatch between model and experimental data is most probably due to a change in constitutive parameters over time.

Conclusions Our results show that the correlation between density and yield stress measurements, as a function of different measuring equipment, strongly depends on the type of mud. Therefore, a proper calibration should be performed by establishing a reference database for each equipment. In order to decrease the amount of in situ survey, it is also necessary to develop models that can accurately predict the consolidation over time.
\end{abstract}

Keywords Fluid mud $\cdot$ Nautical bottom $\cdot$ Predictive maintenance $\cdot$ Shear strength $\cdot$ Yield stress

Responsible editor: Jasmina Obhodas

Alex Kirichek

Alex.Kirichek@deltares.nl

1 Deltares, Boussinesqweg 1, 2629 HV Delft, the Netherlands

2 Faculty of Civil Engineering and Geosciences, Department of Hydraulic Engineering, Delft University of Technology, Stevinweg $1,2628 \mathrm{CN}$ Delft, the Netherlands

3 Department of Chemical, Polymer and Composite Materials Engineering, University of Engineering and Technology, KSK Campus, Lahore 54890, Pakistan

\section{Introduction}

In order to keep the waterways navigable, port authorities have to regularly maintain the silted channels in the port area by means of hopper dredging and relocation of the dredged sediments to the open sea. The intervention protocols in ports and waterways are decided upon bathymetry charts, which includes nautical bottoms, i.e., "the level where physical characteristics of the bottom reach a critical limit beyond which contact with a ship's keel causes either damage or unacceptable effects on controllability and manoeuvrability" (PIANC 2014). The nautical depth should be properly assessed for safeguarding navigation of maritime transport entering the port. 
Traditionally, the surveying in ports is conducted using multibeam echo-sounders. The high-frequency (more than $200 \mathrm{kHz}$ ) multibeam echo-sounders are proven efficient in detecting the water-mud interface (lutocline), which is used as a reference worldwide for a nautical bottom criterion in many ports. However, the multibeam echo-sounding techniques are particularly adapted to water bodies with sharp interfaces like the one existing between a water phase and a sediment bed. Fluid mud layers on the other hand are layers with large vertical density gradients and multibeam echosounding techniques are, therefore, less adapted to detect them. Fluid mud, a mixture of water, organic matter and minerals, is defined by densities ranging from 1030 to $1300 \mathrm{~kg} \mathrm{~m}^{-3}$. Fluid mud layers have typical yield stress values below $20 \mathrm{~Pa}$ (Shakeel et al. 2020 and references within). Mud layers having yield stresses below 20 Pa exhibit very small complex modulus values and strong frequency dependence, which indicated their weak structure (i.e., liquid-like). This type of mud is usually found in estuaries and in rivers with low-intensity currents (Ross and Mehta 1989). This so-called "fluid mud" is in a transient state and settles and consolidates with time, unless mixing energy is added by means of maintenance dredging or natural currents. Since current acoustic methods are not capable to detect the bed in the areas with fluid mud layers, alternative surveying methods have been tested in different ports. The early developed measuring tools (such as radioactive probes) were typically designed to measure mud densities, where mud density was used as a criterion for defining the nautical bottom (e.g., the Port of Rotterdam, the Port of Zeebrugge) (McAnally et al. 2007). Moreover, density is also an important parameter for maintenance dredging because it can be used for estimating the volumes that are required to be dredged.

One of the most prominent drawbacks of these techniques is that they provide only a vertical (density) profile in a water column at a specific location. To get a spatial profile, seismicbased methods (e.g., SILAS) were introduced in order to interpolate the density data over the port area.

Since the early days of fluid mud research, it was recognised that the density is not the only parameter that should be accounted for to assess the nautical bottom (Kirby et al. 1980; Wurpts and Torn 2005; PIANC 2014; Kirichek et al. 2018). Already in 2005, the Port of Emden authorities implemented a criterion based on yield stress measurements as their nautical bottom definition. In their approach, the yield stress of mud samples is found using a conventional rheometer in the laboratory. As usual for rheological measurements, a protocol should be followed in order to assess the rheological properties as the measurements are dependent on the sample history, the type of rheological experiments and the measuring geometries. Currently, several measuring protocols exist in the literature. Wurpts and Torn (2005) used stress sweep tests and Couette geometry. Claeys et al. (2015) proposed another protocol with vane geometry that includes preconditioning of mud. Recently, Shakeel et al. (2020) conducted an extensive laboratory rheological study on mud samples of different densities. Different measuring geometries, rheological methods and protocols were compared. The authors found that a stress sweep test with Couette geometry is a practical and time-efficient method for determining the yield stress values of mud samples with weak yield stress values (Shakeel et al. 2020).

The in situ strength measurements of deposited and fluid mud only became possible in the last decade. One of the tools that provides yield stress is Rheotune. The working principle of the Rheotune is similar to the one of a tuning fork. Rheotune gives vertical profiles of (Bingham) yield stress and density as output. The vertical profiles of density can be interpolated spatially via the SILAS system that links the seismic signal of $38 \mathrm{kHz}$ to the densities measured by Rheotune (Diaferia et al. 2013). The SILAS system is currently used for detection of the nautical bottom in the Port of Rotterdam and in the Port of IJmuiden.

Another tool that gives information about the strength of mud beds is the Graviprobe (Bezuijen et al. 2018). The Graviprobe is a free-fall cylinder that penetrates the watermud column under its own weight. The primary measurement of the Graviprobe is acceleration/deceleration of the cylinder. The measurements are related to an undrained shear strength using a force balance equation. The undrained shear strength is not a rheological parameter, but it can be used for determining the strength of mud layers. The Graviprobe is currently tested in the Port of Rotterdam.

The purpose of the present study is to compare the applicability of density, shear strength and yield stress criteria with the definition of nautical bottom. Rheotune and Graviprobe were tested under different circumstances in situ in different areas of the Port of Hamburg and at the Port of Rotterdam. The in situ measurements were then compared with yield stress and density measurements carried out in the laboratory. In the last part, densities and undrained shear strength measurements are presented as a function of time and used to predict the development of strength over time.

\section{Methods}

Figure 1 shows the in situ tools that were used in this research. The Graviprobe (from DotOcean) measures the acceleration/ deceleration while falling freely in a water-mud column. The undrained shear strength is then obtained from the acceleration/deceleration using a force balance equation (Bezuijen et al. 2018). The Rheotune (from Stema Systems) records the amplitudes which are triggered by mechanical vibrations at the frequency range of $500-800 \mathrm{~Hz}$. The recordings can then be used to get the information about the 
Fig. 1 In situ measuring devices used in the study

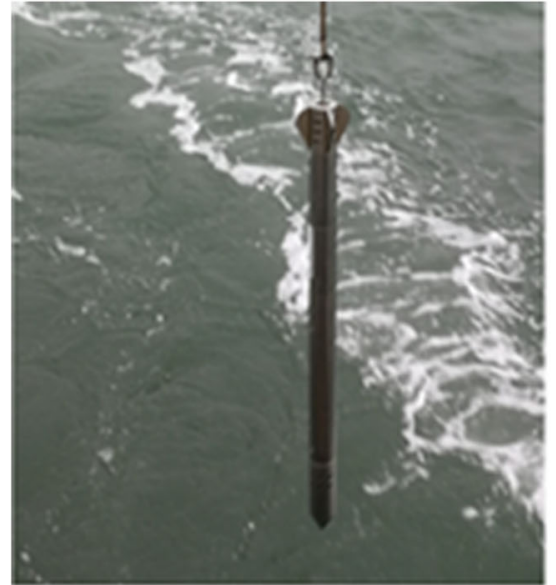

Graviprobe

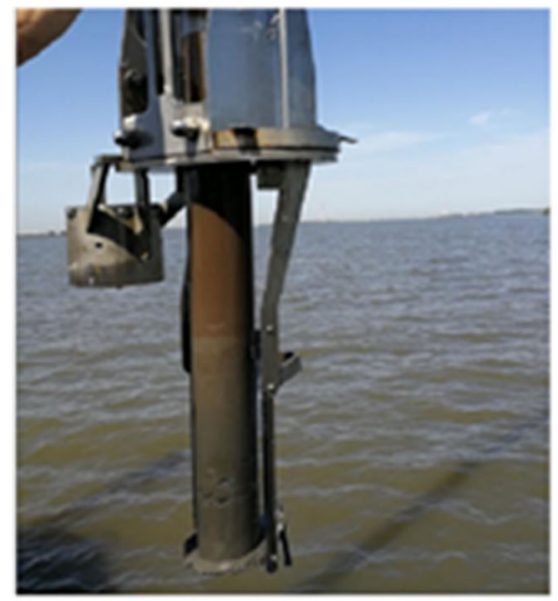

Frahmlot

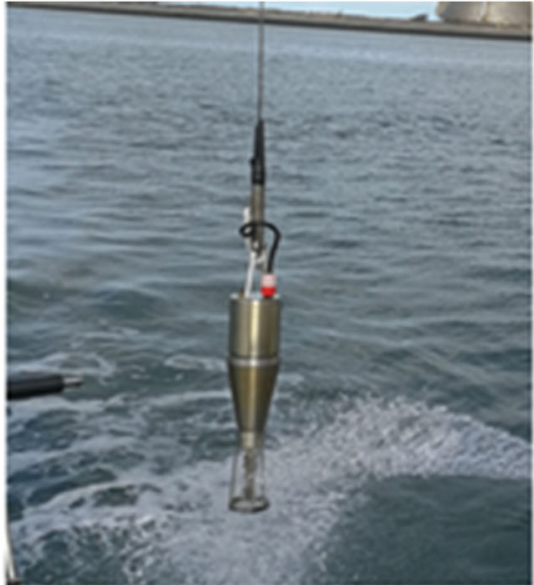

Rheotune

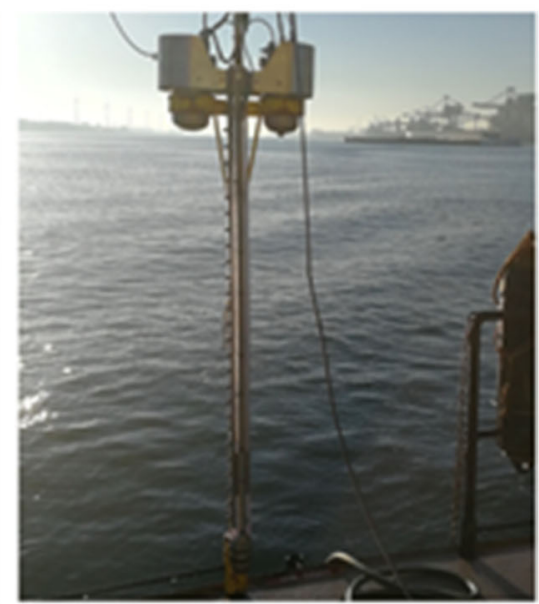

Slibsampler
(Bingham) yield stress and density of mud layers (Fonseca et al. 2019).

A so-called Frahmlot is used to collect mud samples. This sampler allows to collect cylindrical core samples of mud of $1 \mathrm{~m}$, which are then manually divided in smaller samples. The samples collected with the Frahmlot were analysed in the laboratory. Both the density and the yield stress of the samples were measured. The yield stress was assessed using a recently developed rheological protocol (Shakeel et al. 2019, 2020). A HAAKE MARS I rheometer (Thermo Scientific, Germany) was used to perform the rheological experiments with concentric cylinder (Couette) geometry.

Four different locations at the Port of Hamburg were used to test the Rheotune and to compare the results with laboratory measurements: Vorhafen (VH), Köhlfleet mit Köhlfleethafen (KH), Rethe (RT) and Reiherstieg Vorhafen (RV) (see Fig. 2 for selected locations). Sedimentfang Wedel (SW) (another location in Port of Hamburg, see Fig. 2) was used to compare the measurements of the Graviprobe and Rheotune on 07-062018, 28-06-2018, 07-08-2018 and 18-09-2018. A previous analysis revealed the existence of significantly different rheological properties for mud samples collected from the selected locations. These differences are due to different densities and organic matter content (Shakeel et al. 2020).

Graviprobe and Rheotune measurements were also compared in a sediment trap in the Calandkanaal at the Port of Rotterdam (Fig. 2b). The sediment trap was originally about $1.5 \mathrm{~m}$ deeper than the natural bed in the Calandkanaal. Water injection dredging activities were performed in order to fluidise the mud layer in the Calandkanaal. Subsequently, the fluidised mud was advected into the sediment trap. Regular Graviprobe and Rheotune surveys were carried out after the water injection dredging in the sediment trap. More information on the pilot at the Port of Rotterdam is given in Kirichek and Rutgers (2019).

The combination of a "Slibsampler" and a DMA 35 Anton Paar density meter was used to measure the density along the collected core samples. The Slibsampler is a cylindrical core sampling device with a diameter of $5 \mathrm{~cm}$. Inlets are installed along a lateral surface of the core (see Fig. 1), so that the fluid mud can be subsampled from the collected core using a syringe. The subsample was flashed 
a)

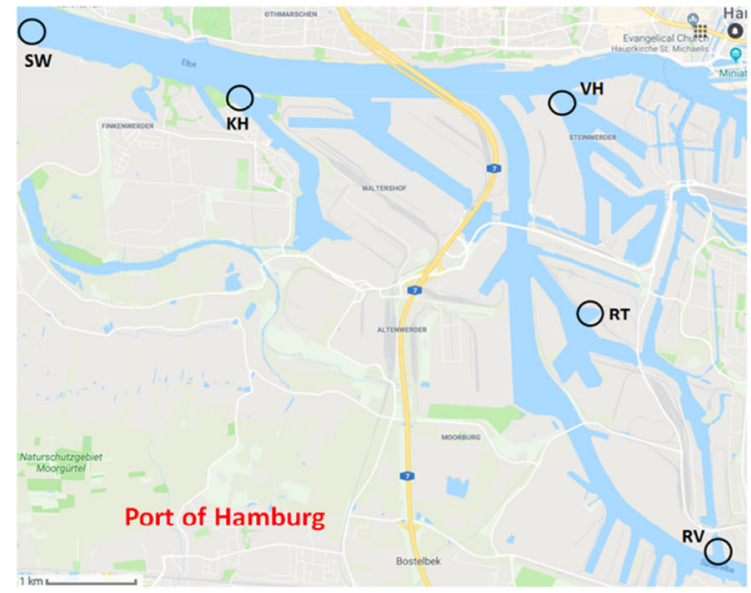

b)

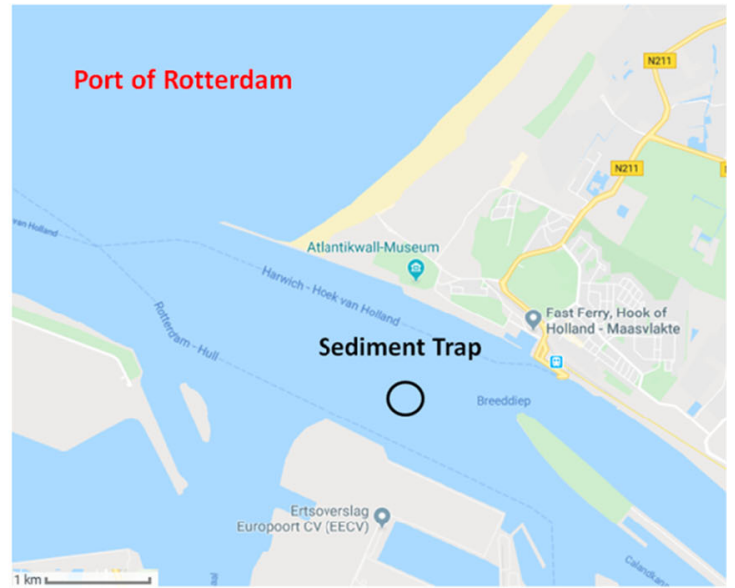

Fig. 2 Selected locations in the a Port of Hamburg, Germany, and b Port of Rotterdam, the Netherlands, to collect mud samples

through the Anton Paar density meter which gives the density with an error of $1 \mathrm{~kg} \mathrm{~m}^{-3}$. This procedure is repeated for each inlet and hence a vertical in situ density profile of mud can be obtained.

A model that describes the consolidation of weak mud was used to relate the strength and density measurements. This model was validated for different natural mud samples from Ems-Dollard Estuary and the Caland-Beer Channel at the Port of Rotterdam (Merckelbach 2000).

\section{Results and discussion}

Figure 3 shows the yield stresses measured in situ and in laboratory as a function of density at different locations of the Port of Hamburg. The in situ measurements for Vorhafen (VH) and for Köhlfleet mit Köhlfleethafen (KH) match well with the laboratory experiments. However, the values measured for Rethe (RT) and Reiherstieg Vorhafen (RV) showed the smaller in situ Bingham yield stress compared with the one
Fig. 3 Comparison of densities and yield stresses measured in situ using the Rheotune and in laboratory using the Couette rheometer. The abbreviations indicate different locations in the Port of Hamburg: Vorhafen (VH), Köhlfleet mit Köhlfleethafen (KH), Rethe (RT) and Reiherstieg Vorhafen (RV)
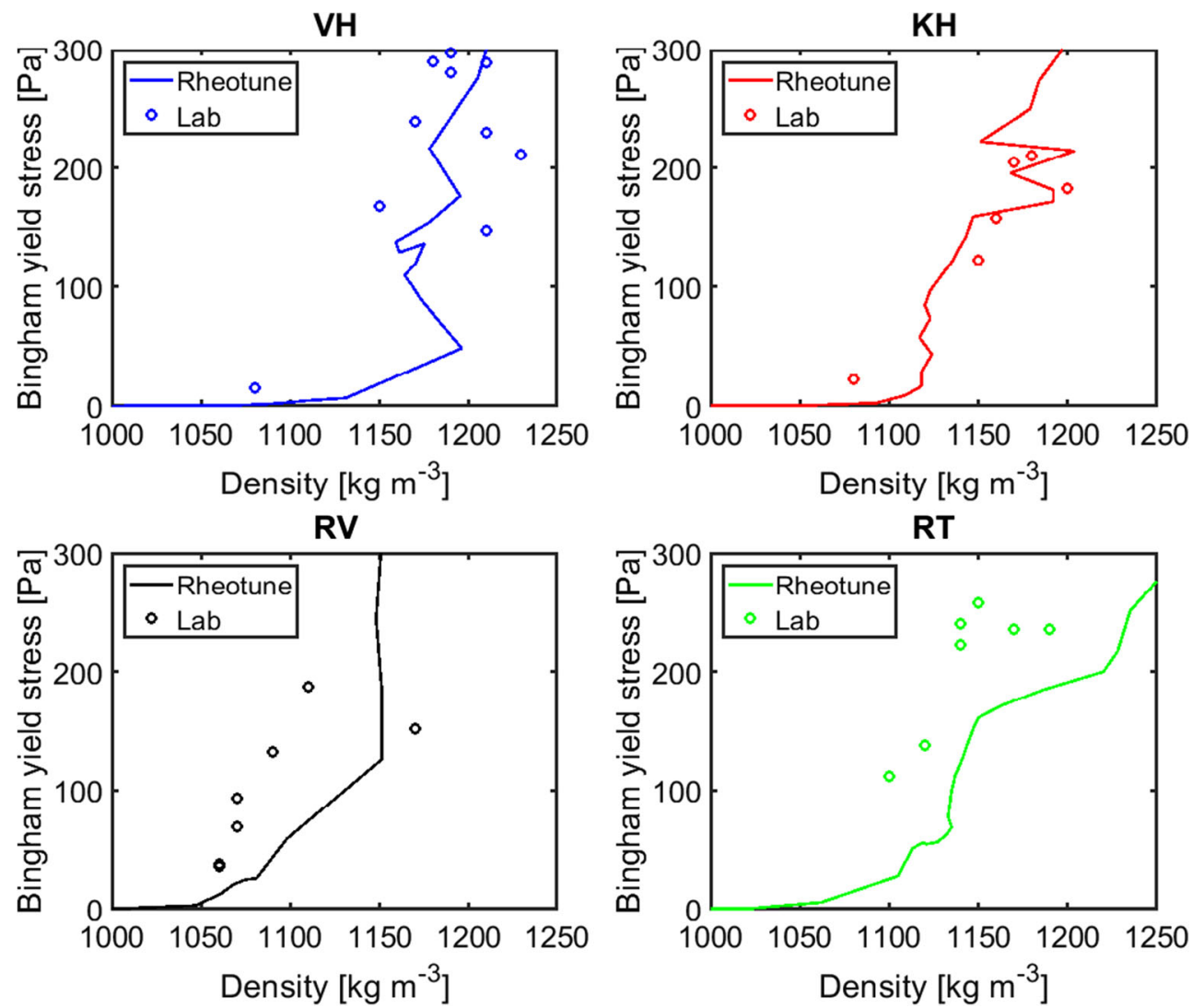

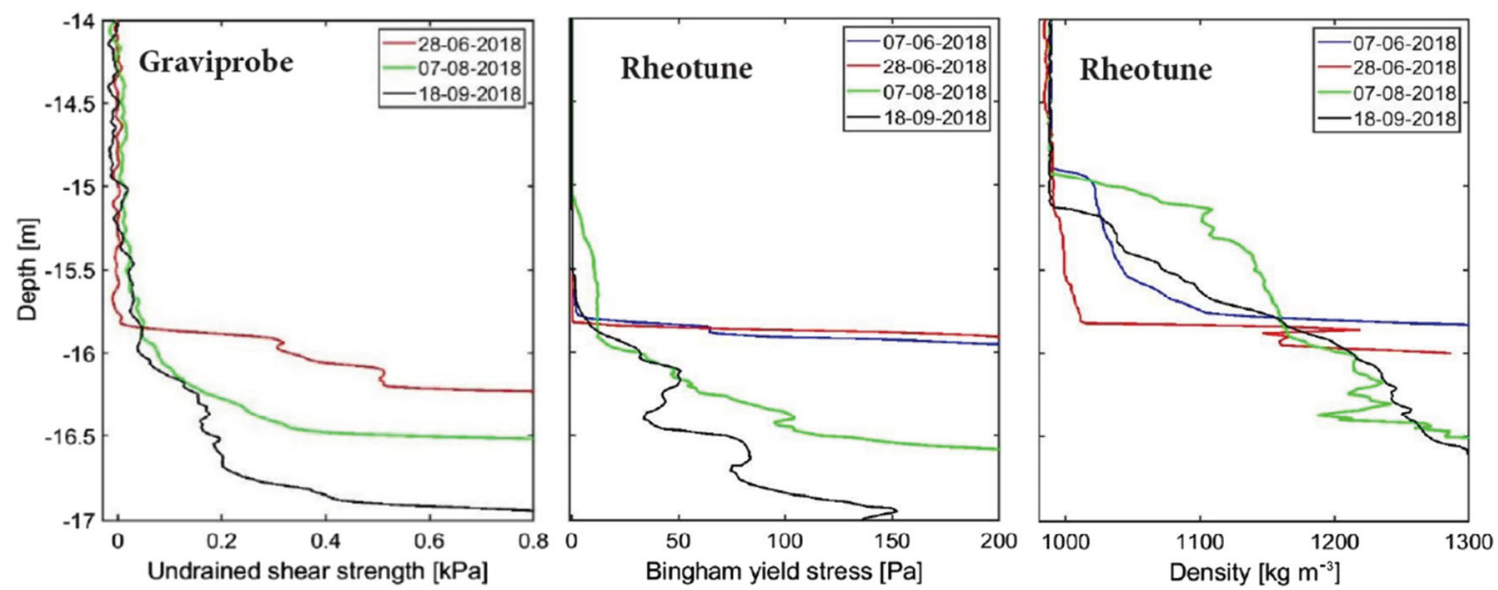

Fig. 4 Comparison of Rheotune (yield stress and density) and Graviprobe measurements (undrained shear strength) in the Port of Hamburg (Sedimentfang Wedel). The Rheotune (density) plot shows sedimentation before and after dredging shortly after 28-06-2018

measured in the laboratory. The discrepancy can be due to high organic matter content in RT and RV (Shakeel et al. 2020).

The comparison between the Graviprobe's and Rheotune's profiles is shown in Fig. 4. The thickness of the fluid mud layer, found from each device, is similar. The evolution of the undrained shear strength and Bingham yield stress followed the same trend in time. The measurements conducted on 18-09-2018 show that weak fluid mud layers can be measured by both Graviprobe and Rheotune. On 18-09-2018, this weak fluid mud layer had an undrained shear strength smaller than $0.2 \mathrm{kPa}$ and a thickness of $1.25 \mathrm{~m}$. We note that undrained shear strength and yield stress have a non-linear dependence (Bezuijen et al. 2018). One important upcoming study will be to analyse the relation between these properties.

The Rheotune measurements report a weak mud layer thickness $(<100 \mathrm{~Pa})$ of about $1.20 \mathrm{~m}$. The measurements conducted by Graviprobe and Rheotune (Bingham yield stress) on 07-08-2018 and on 28-06-2018 also show an acceptable correspondence. The corresponding density measurements are given on the right panel of Fig. 4.

The Rheotune and the Graviprobe have also been tested in the Port of Rotterdam. Figure 5 shows the corresponding densities and yield stress measurements from Rheotune. The density was estimated using (a) the Rheotune and (b) the Anton Paar density meter combined with the Slibsampler. The yield stress was measured by laboratory rheological analysis. It is found that the Rheotune and Slibsampler density data are in very good agreement, as is the Bingham yield stress data found by Rheotune and rheological measurements in the lab. The reason for the better agreement, than for the experiments conducted in the Port of Hamburg, is most probably related to the lower amounts of organic matter content in the Port of Rotterdam.

In the last part, it was checked whether modelling could reproduce the in situ strength and density development as a function of time. The comparison between model and experimental data was done for the measurements conducted in the
Fig. 5 Validation of density and yield stress values measured by Rheotune in the Port of Rotterdam at the sediment trap after water injection dredging. Wk02 = after 2 weeks, wk04 = after 4 weeks, wk07 $=$ after 7 weeks and wk08 $=$ after 8 weeks
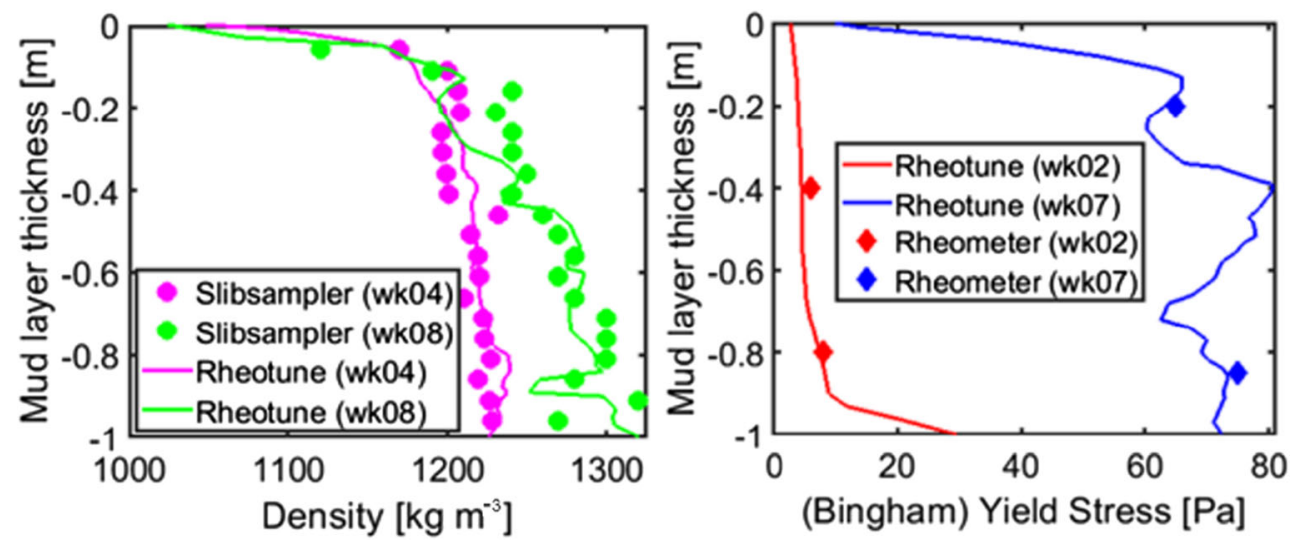
sediment trap at the Port of Rotterdam, see Fig. 6. The hydrodynamic conditions in the trap are expected to be very low-a prerequisite for the model. The numerical code solves the Gibson equation (Merckelbach 2000) and requires three input parameters: the initial mud density as a function of depth, and the two material parameters: the permeability $K$ and the effective stress $\sigma$ function of density $\rho$. For last two parameters, following the work of Merckelbach (2000), we took $K=K_{\mathrm{k}}$ $\cdot \rho^{\mathrm{n}}$ and $\sigma=K_{\text {sigma }} \cdot \rho^{\mathrm{n}}$. For $n$, we used 6.67 , which is a number in line with the findings of Merckelbach (2000). $K_{\mathrm{k}}$ and $K_{\text {sigma }}$ are constants to be calibrated. We found $K_{\mathrm{k}}=7 \cdot 10^{-13}$ and $K_{\text {sigma }}=7 \cdot 10^{7}$. The model's output is the mud density as a function of time and depth. The measured density profiles show a good resemblance with the modelling, but differences are observed. The strength measurements are in line with the modelling results of the effective stresses. The observed differences can be due to several reasons: (1) the hydrodynamic activity in the trap, even though low, can contribute to a change in density and strength in time and space, (2) The accuracy of the in situ data poses a problem. It is in particular very difficult to sample twice at the exact same location, (3) the constitutive parameters (permeability and effective stress) are taken to be constant in the model and adjusted to reproduce the data as best as possible. However, these parameters could be changing over time, as the properties of mud are evolving due to its composition and complex interaction with its environment.

\section{Conclusions}

Recent progress in the development of the in situ shear strength and rheology measuring equipment enables to use these methods in waterways with fluid mud layers to assess the properties of mud layers. In the present work, we reported the results obtained using the Rheotune and Graviprobe, to assess respectively (in situ) the density and yield stress (Rheotune) and the undrained shear strength (Graviprobe) of mud layers in the different areas of the Port of Hamburg and the Port of Rotterdam. Our study shows that the density measurements using Rheotune match well with density values measured using an Anton Paar density meter. The yield stress values found using the Rheotune correlates well with the yield stresses measured in the laboratory for two locations but not for two others, where the in situ Bingham yield stresses are systematically lower than the ones found in the laboratory. This finding is attributed to the diversity and amount of organic matter in the harbour.

The measurements of Graviprobe and Rheotune, density and strength profiles, are in good agreement with the settling and consolidation model presented in the article, which was developed for mud layers at the early stages of consolidation. In this study, $K_{\mathrm{k}}$ and $K_{\text {sigma }}$ were used as (constant) adjustable parameters for the model; however, these parameters can also be obtained in laboratory by performing specific experiments on the mud (Merckelbach 2000). Further research is required
Fig. 6 Modelled and measured water-mud interface (a), density (b) and strength (c) at the Port of Rotterdam. The measurements are conducted after water injection dredging actions. For the model, we used an initial density of $1123 \mathrm{~kg} \mathrm{~m}^{-3}$ and a mud height of $2 \mathrm{~m}$
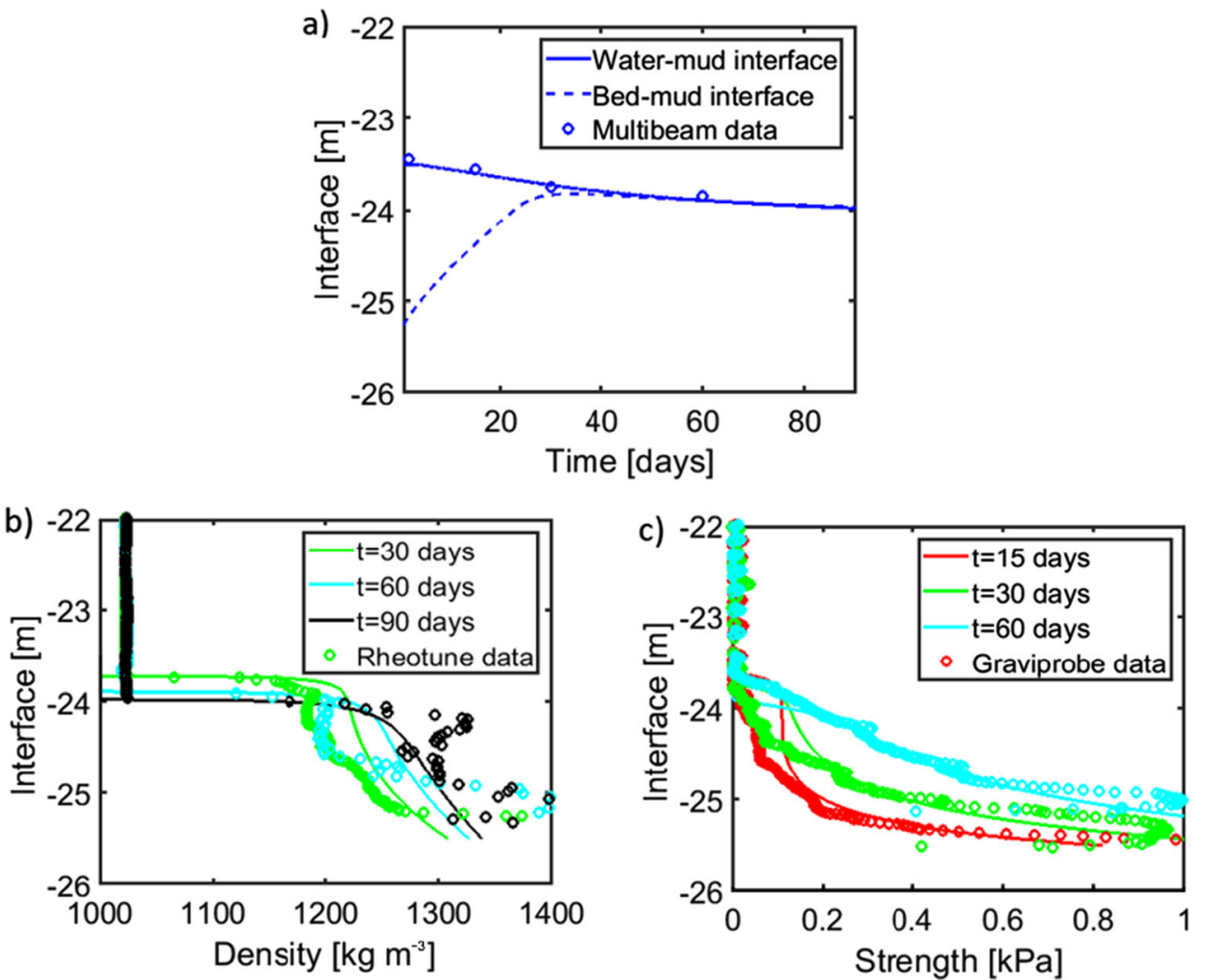
to study if laboratory-obtained $K_{\mathrm{k}}$ and $K_{\text {sigma }}$ are in line with the adjusted $K_{\mathrm{k}}$ and $K_{\text {sigma }}$ used to fit the in situ data.

Data availability This study is carried out within the framework of the MUDNET academic network https://www.tudelft.nl/mudnet/.

\section{Compliance with ethical standards}

Conflict of interest The authors declare that they have no conflict of interest.

\section{Human participant and/or animal rights and informed consent None.}

Open Access This article is licensed under a Creative Commons Attribution 4.0 International License, which permits use, sharing, adaptation, distribution and reproduction in any medium or format, as long as you give appropriate credit to the original author(s) and the source, provide a link to the Creative Commons licence, and indicate if changes were made. The images or other third party material in this article are included in the article's Creative Commons licence, unless indicated otherwise in a credit line to the material. If material is not included in the article's Creative Commons licence and your intended use is not permitted by statutory regulation or exceeds the permitted use, you will need to obtain permission directly from the copyright holder. To view a copy of this licence, visit http://creativecommons.org/licenses/by/4.0/.

\section{References}

Bezuijen A, den Hamer DA, Vincke L, Geirnaert K (2018) Free fall cone tests in kaolin clay. Physical Modelling in Geotechnics, Volume 1, Proceedings of the 9th International Conference on Physical Modelling in Geotechnics (ICPMG 2018)

Claeys S, Staelens P, Vanlede J, Heredia M, Van Hoestenberghe T, Van Oyen T, Toorman E (2015) A rheological lab measurement protocol for cohesive sediment. Book of abstracts: 13th International Conference on Cohesive Sediment Transport Processes

Diaferia G, Kruiver PP, Vermaas T (2013) Validation study of SILAS, Final report, Deltares, Delft the Netherlands, 1207624-000-BGS0006

Fonseca DL, Marroig PC, Carneiro JC, Gallo MN, Vinzón SB (2019) Assessing rheological properties of fluid mud samples through tuning fork data. Ocean Dynam 69(1):51-57

Kirby R, Parker WR, van Oostrum WHA (1980) Definition of the seabed in navigational routes through mud areas, international hydrographic review, Monaco. LVII 1

Kirichek A, Rutgers R (2019) Water injection dredging and fluid mud trapping pilot in the port of Rotterdam, Proceedings for CEDA Dredging Days, Rotterdam, the Netherlands

Kirichek A, Chassagne C, Winterwerp H, Vellinga T (2018) How navigable are fluid mud layers? Terra Aqua 151:6-18

McAnally WH, Friedrichs C, Hamilton D, Hayter E, Shrestha P, Rodriguez H, Sheremet A, Teeter A (2007) Management of fluid mud in estuaries, bays, and lakes. : Present state of understanding on character and behavior. I J Hydraulic Eng 133:9-22

Merckelbach LM (2000) Consolidation and strength evolution of soft mud layers. Dissertation, Delft University of Technology

PIANC (2014) Harbour approach channels - design guidelines, 121

Ross MA, Mehta AJ (1989) On the mechanics of Lutoclines and fluid mud. J Coast Res 5:51-62

Shakeel A, Kirichek A, Chassagne C (2019) Is density enough to predict the rheology of natural sediments? Geo-Marine Lett 39:427-434

Shakeel A, Kirichek A, Chassagne C (2020) Rheological analysis of mud from port of Hamburg, Germany. J Soils Sediments 19:1-10. https:// doi.org/10.1007/s11368-019-02448-7

Wurpts R, Torn P (2005) 15 years' experience with fluid mud: definition of the nautical bottom with rheological parameters. Terra et Aqua 99:22-32

Publisher's note Springer Nature remains neutral with regard to jurisdictional claims in published maps and institutional affiliations. 